\title{
Funktionelle Sehschärfe beim Trockenen Auge
}

\author{
Elisabeth M. Messmer (D)
}

Eingegangen: 8. März 2021 / Angenommen: 22. April 2021 / Online publiziert: 9. Juni 2021

(c) Der/die Autor(en) 2021

Zusammenfassung Das Trockene Auge führt häufig zu Sehstörungen trotz guter Sehleistung in der Standardvisusprüfung. Die Beschwerden treten meist beim Lesen, Arbeiten am Computer und/oder beim Autofahren auf. Grund ist die schlechte optische Qualität der Augenoberfläche beim Trockenen Auge mit Zunahme von topometrischer Irregularität und Asymmetrie, Anstieg des irregulären Astigmatismus, Zunahme der kornealen und okulären Aberrationen höherer Ordnung, Abnahme der Kontrastsensitivität und Zunahme der Lichtstreuung. Die funktionelle Sehschärfe repräsentiert die Visusfunktion im Zeitverlauf und reflektiert die Visusleistung eines Individuums bei täglichen Aktivitäten. Sie ist beim Trockenen Auge signifikant reduziert im Vergleich zu Normalprobanden. Eine Vielzahl von Testmethoden zur Evaluierung der funktionellen Sehschärfe wurde beschrieben. Kein Verfahren hat sich bisher in der täglichen Praxis durchgesetzt.

Schlüsselwörter Trockenes Auge · Funktionelle Sehschärfe · Optische Qualität · Kontrastsensitivität · Aberrationen höherer Ordnung

\section{Functional visual acuity in dry eye disease}

Summary Dry eye disease (DED) often leads to visual disturbances despite good visual acuity on standard vision testing. Symptoms mainly occur when reading, working on a computer and/or driving a car. The inferior optical quality of the ocular surface in DED seems to be responsible for these symptoms, with an increase of topometric irregularity and asymmetry,

Prof. Dr. med. E. M. Messmer (凶)

Augenklinik, Ludwig-Maximilians-Universität München,

Mathildenstr. 8, 80336 München, Deutschland

emessmer@med.uni-muenchen.de rise in irregular astigmatism, increase in corneal and ocular higher order aberrations, decrease in contrast sensitivity and increase in light scattering. Functional visual acuity represents the visual function over time and reflects the visual performance of an individual during daily activities. It is significantly reduced in DED compared to controls. A number of tests have been reported for evaluation of functional visual acuity. No single method has so far become established in the daily clinical practice.

Keywords Dry eye disease · Functional visual acuity · Optic quality · Contrast sensitivity · Higher order aberrations

Das Trockene Auge wurde im Dry Eye Workshop (DEWS) I im Jahr 2007 u. a. definiert als „multifaktorielle Erkrankung der Tränen und der Augenoberfläche, die subjektiven Dyskomfort, Sehstörungen, Tränenfilminstabilität und eine mögliche Schädigung der Augenoberfläche verursacht" [1]. Somit wurde den vom Patienten häufig beklagten Sehstörungen beim Trockenen Auge Rechnung getragen. Leider wurden in der revidierten Definition des DEWS II von 2017 diese sehr häufig geäußerten Sehstörungen nicht mehr in die Definition aufgenommen, es wird hier nur noch generell von „okulären Symptomen“ gesprochen [2].

\section{Optische Qualität beim Trockenen Auge}

Die optische Qualität beim Trockenen Auge ist schlecht (Abb. 1). Bei verlängerter Augenöffnung kommt es bei Patienten mit Trockenem Auge zu einer signifikanten Zunahme des okulären „surface regularity index“ (SRI) in der Hornhauttopometrie als Ausdruck einer zunehmenden Irregularität der Hornhautoberfläche [3]. Fluktuationen und Unregelmäßigkeiten des Trä- 
Abb. 1 Optische Qualität beim Trockenen Auge
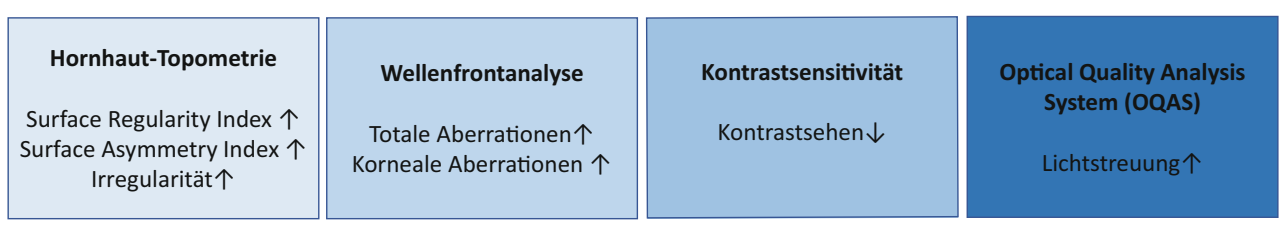

Abb. 2 Sehstörungen beim Trockenen Auge
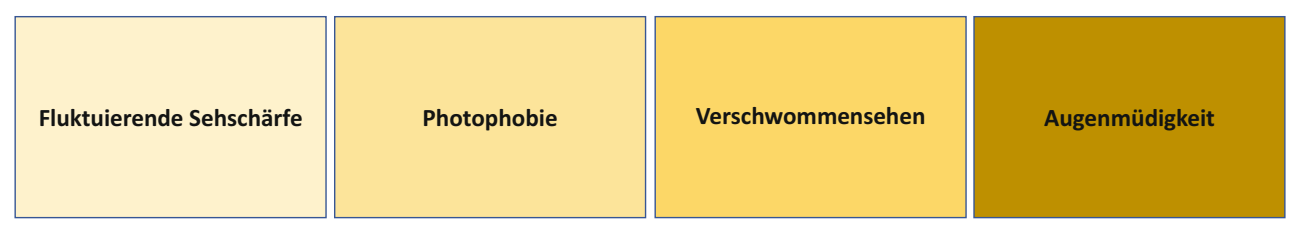

nenfilms bei Trockenem Auge erhöhen zudem den „surface asymmetry index“ (SAI) und den irregulären Astigmatismus insgesamt [4]. Sequenzielle Wellenfrontanalysen der Hornhaut dokumentierten die Verschlechterung der optischen Qualität nach einem Lidschlag mit Zunahme der kornealen und totalen Aberrationen höherer Ordnung [4-6]. Diese Zunahme der Aberrationen höherer Ordnung wird auch bei Trockenem Auge ohne Hornhautschädigung z. B. im Rahmen eines „Short BUT-Dry Eye“ gesehen [7, 8]. Auch die Vorwärtslichtstreuung („forward light scattering“) ist beim Trockenen Auge signifikant erhöht [7]. Zudem ist die Kontrastsensitivität bei Patienten mit Trockenem Auge erniedrigt [9, 10]. Diese optischen Phänomene erklären die schwankende Sehschärfe nach einem Lidschlag beim Trockenen Auge. Das simulierte Netzhautbild beim Trockenen Auge ist sofort nach dem Lidschlag unscharf und bleibt es zwischen den Lidschlägen [7]. Eine kompensatorische Zunahme der Lidschläge ist die Folge [9]. Allerdings sind diese Lidschläge häufig inkomplett und somit relativ wirkungslos.

\section{Visuelle Beschwerden beim Trockenen Auge}

Patienten mit mildem bis moderatem Trockenem Auge attestieren bei der Standardvisusprüfung meist eine regelrechte bestkorrigierte Sehschärfe. Trotzdem klagen sie über Sehstörungen, fluktuierende Sehschärfe, Verschwommensehen, Photophobie und Augenmüdigkeit ([7, 9, 11]; Abb. 2). Diese Beschwerden treten meist bei bestimmten Tätigkeiten wie Lesen, Arbeiten am Computer oder Autofahren auf, schränken oft die Lebensqualität der Patienten ein und können $\mathrm{zu}$ verminderter Leistung sowohl in der Arbeit als auch in der Freizeit führen [11]. Ansteigend mit dem Schweregrad der Erkrankung gaben Patienten mit Trockenem Auge in einer aktuellen Studie Einschränkungen der visuellen Funktion im Ocular Surface Disease Index(OSDI)-Fragebogen an. Besonders das Lesen, das Autofahren bei Nacht, Computerarbeit und Fernsehen waren hier eingeschränkt [12]. Tageszeitliche Schwankungen scheinen zudem die visuellen Phänomene beim Trockenen Auge zu beeinflussen. Walker et al. zeigten, dass am Abend die bestkor- rigierte Sehschärfe zwischen 2 Lidschlägen weniger stabil und die Lesezeiten für einen bestimmten Text verlängert waren im Vergleich zu morgens [13].

Die Zeit des Lidschlusses/min ist bei Patienten mit Trockenem Auge mit 4,5\% zudem signifikant länger als in der Normalbevölkerung mit 0,7\%. Die Anzahl überlanger Lidschlusszeiten von $>0,5 \mathrm{~s}$ ist darüber hinaus signifikant höher und mit einem signifikant verringerten Intervall zwischen den Lidschlägen verbunden [14].

Gerade im Rahmen von ophthalmologischen Eingriffen wie hornhautrefraktiver Chirurgie oder Kataraktoperation spielen visuelle Symptome im Rahmen eines Trockenen Auges eine zunehmende Rolle und können Ursache für eine fehlerhafte Intraokularlinsenberechnung und eine postoperative Patientenunzufriedenheit sein [15-18].

\section{Lesen mit Trockenem Auge}

Viele Patienten mit Trockenem Auge klagen über Probleme beim Lesen. Karakus et al. beschrieben eine klinisch signifikant langsamere Lesegeschwindigkeit bei längerer Lesetätigkeit bei Patienten mit Trockenem Auge verglichen mit Kontrollen. Die Reduktion visusrelevanter Unterpunkte im OSDI-Fragebogen sowie eine zunehmende Hornhautanfärbung waren mit der Reduktion der Lesegeschwindigkeit signifikant korreliert [19, 20]. Während einer 30-minütigen Lesephase verschlechterten sich multiple Parameter des Trockenen Auges wie Bindehaut- und Hornhautanfärbung, Tränenfilmaufreißzeit, Schirmer-Test und der Surface Regularity Index in der Topographie. Umso niedriger die Tränenfilmaufreißzeit und der Schirmer-Test vor dem Lesen waren, umso gravierender veränderten sich diese klinischen Zeichen nach dem Lesen [21]. Mathews et al. fanden eine signifikant langsamere, um $14 \%$ reduzierte Lesegeschwindigkeit sowohl beim lauten Vorlesen als auch beim stillen Lesen beim Trockenen Auge im Vergleich zu Kontrollen. Die langsamere Lesegeschwindigkeit korrelierte zum subjektiven Beschwerdescore im OSDI-Fragebogen und zu wortspezifischen Merkmalen (längere und selten gebrauchte Wörter) [22]. Längeres Lesen könnte ein klinisch relevanter objektiver Test sein, um den 
Einfluss des Trockenen Auges auf die Visus-assoziierte Lebensqualität zu testen, die Diskordanz zwischen Symptomen und Befunden $\mathrm{zu}$ verstehen und neue Therapien zu evaluieren [19-22].

\section{Autofahren mit Trockenem Auge}

Viele Patienten mit Trockenem Auge klagen über Probleme beim Autofahren. Verstärkt werden diese Probleme durch schlechte Lichtverhältnisse, blendendes Sonnenlicht und die Klimaanlage im Auto.

Deschamps et al. untersuchten 20 Patienten mit Trockenem Auge und 20 Kontrollprobanden in einem speziellen Fahrsimulator. Die visusabhängige Fahrfähigkeit wurde durch das Erkennen von zufallsbedingt projizierten Zielobjekten mit zunehmendem Kontrast getestet. Es wurden die Anzahl der gesehenen Objekte, ihre Position sowie die Reaktionszeit registriert. Zusätzlich wurden die Studienteilnehmer klinisch untersucht, ihre subjektiven Trockenen Augen-Symptome mit dem OSDI-Fragebogen erfasst sowie Aberrationen höherer Ordnung gemessen. Die Anzahl an verpassten Zielobjekten und die Reaktionszeit waren signifikant größer bei Patienten mit Trockenem Auge im Vergleich zu Kontrollen. Insbesondere in spezifischen Verkehrssituationen wie Kreuzungen und Kreisverkehr war die Sehfunktion von Patienten mit Trockenem Auge eingeschränkt. Die Reaktionszeit korrelierte positiv mit dem Progressionsindex für Aberrationen höherer Ordnung und mit den subjektiven Symptomen im OSDI-Fragebogen. Diese Studie bestätigte objektiv und eindrücklich die von den Patienten geäußerten Beschwerden beim Autofahren [23].

\section{Konzept der funktionellen Sehschärfe}

Die funktionelle Sehschärfe repräsentiert die Visusfunktion im Zeitverlauf. Sie reflektiert die Visusleistung eines Individuums bei gewissen täglichen Aktivitäten, die das Sehen erfordern. Sie wird gemessen bei über 10-20s geöffnetem Auge ohne Lidschlag. Das Konzept fundiert auf der Erkenntnis, dass es beim Autofahren, Lesen oder Arbeiten am Computer zu Symptomen wie Verschwommensehen und/oder Augenmüdigkeit kommt [24]. Ein intakter Tränenfilm ist ein kritischer Faktor für die Stabilität der optischen Qualität der Augenoberfläche und relevant für ein qualitativ hochwertiges retinales Bild. Wenn der Lidschlag beim Sehen unterdrückt wird, trocknet die Augenoberfläche aus. Patienten haben dann Schwierigkeiten, ein klares Bild aufrechtzuerhalten. Die sog. „visual maintenance ratio“ (VMR) ist das Verhältnis von funktioneller Sehschärfe und der bestkorrigierten Basissehschärfe. Sowohl die funktionelle Sehschärfe als auch die VMR sind Indizes für die visuelle Funktion [25]. Die Prüfung der funktionellen Sehschärfe deckt Sehfunktionsstörungen auf, die mit der konventionellen Sehschärfenprüfung nicht erkannt werden.
Die funktionelle Sehschärfe ist beim Trockenen Auge reduziert. Sie korreliert mit klinischen Parametern wie der Tränenquantität, der Tränenstabilität und der Oberflächenanfärbung [26, 27]. Die Messung der funktionellen Sehschärfe wurde auch als Screeninginstrument für das Trockene Auge erfolgreich eingesetzt [28]. Die Analyse der funktionellen Sehschärfe spielt eine zunehmende Rolle auch bei anderen ophthalmologischen Erkrankungen (Glaukom, Katarakt, retinale Erkrankungen, Amblyopie, Presbyopie), vor und nach augenärztlichen Eingriffen (refraktive Chirurgie, Kataraktchirurgie) sowie bei Kontaktlinsenträgern [24].

\section{Untersuchungen zur Messung der funktionellen Sehschärfe und/oder optischen Qualität beim Trockenen Auge}

Es gibt einen dringenden Bedarf, die dynamische funktionelle Sehschärfe und/oder die optische Qualität in der Diagnose und Therapie des Trockenen Auges objektiv zu messen, um visuelle Beschwerden von Patienten besser zu erfassen und neue Therapieoptionen zu evaluieren. Ein Fragebogen zur visuellen Qualität zeigte zuverlässige Werte, die mit Visuswerten, Kontrastsensitivität und Aberrationen höherer Ordnung korrelierten [29]. Eine Reihe von Testmethoden wurde beschrieben. Während einige Methoden die funktionelle Sehschärfe durch aktives Erkennen von Lesezeichen bestimmen (Kontrastsensitivität, IVAD, FVA, FVAM) prüfen andere eher die optische Qualität des visuellen Systems (Aberrationen höherer Ordnung, Tear Stability Analysis System, OQAS-System). Keines der Verfahren hat sich bisher in der täglichen Praxis durchgesetzt.

\section{Aktive Prüfung der funktionellen Sehschärfe}

Kontrastsensitivität, Kontrastsensitivität bei Gegenlicht Eine Vielzahl von Augenerkrankungen wird mit einer reduzierten Kontrastsensitivität bei regelrechter Sehschärfe assoziiert. Rolando et al. beschrieben eine signifikante Reduktion der Kontrastsensitivität bei Trockenem Auge ohne und mit Keratopathie von 35-70\%. Die Kontrastsensitivität verbesserte sich signifikant $15 \mathrm{~min}$ nach Applikation eines Hyaluronhaltigen Tränenersatzmittels [10]. Eine stärker ausgeprägte Meibom-Drüsen-Dysfunktion und eine größere Tränenfilminstabilität waren in einer weiteren Studie mit einer signifikant schlechteren Kontrastsensitivität assoziiert im Vergleich zu Kontrollen [30]. Die visuelle Qualität ist jedoch nicht nur durch die Sensitivität für Kontrast bestimmt. Störende Blendung, die Reduktion von Sehschärfe oder Kontrastwahrnehmung durch eine benachbarte Blendquelle, beeinflusst viele unserer täglichen Aktivitäten, wie z. B. das Autofahren bei Nacht [31]. Puell et al. zeigten, dass die Kontrastsensitivität nicht nur bei regelrechten Lichtverhältnissen bei Patienten mit Trockenem Auge herabgesetzt ist, sondern v. a. bei Gegenlicht 
[31]. Dies erklärt zum Teil die beklagten Beschwerden unserer Patienten beim Autofahren bei Nacht.

IVAD (Inter-Blink-VA-Decay - Reduktion des Visus zwischen 2 Lidschlägen)

Der Inter-Blink-VA-Decay(IVAD)-Test ist ein Maß für den Abfall der Sehfunktion zwischen 2 Lidschlägen. Nach Evaluierung der bestkorrigierten Sehschärfe wird die Leserate einer Liste mit 16 Wörtern monokular getestet. Die Lidschlagrate wird parallel erfasst. Die Zeit zum Verlust einer Visuszeile des bestkorrigierten Visus wird gemessen. Normalprobanden sind in der Lage, die bestkorrigierte Sehschärfe signifikant länger zu halten als Patienten mit Trockenem Auge [32]. Nach Applikation von Tränenersatz verbesserten sich die Werte im IVAD-Test signifikant [32].

\section{FVA-Tester, FVAM-System}

Goto et al. entwickelten erstmalig ein Gerät zur Messung der funktionellen Sehschärfe bei verlängerter Augenöffnung und Unterdrückung des Lidschlags von 10-20 s, den FVA-Tester (Functional-Visual-Acuity-Tester). Allerdings wurde der funktionelle Visus bei anästhesierter Hornhaut unter sehr artifiziellen Bedingungen gemessen. Bei Patienten mit Trockenem Auge zeigt sich die funktionelle Sehschärfe im Vergleich zur bestkorrigierten Sehschärfe signifikant erniedrigt. Bei Normalprobanden waren funktionelle und bestkorrigierte Sehschärfe nicht signifikant unterschiedlich [3]. Beim FVA-System (SSC-350, Nidek, Aichi, Japan) werden dem Patienten automatisch Landolt-Ringe auf einem Bildschirm präsentiert, deren Orientierung erkannt werden muss. Die funktionelle Sehschärfe wurde nach 10, 20 und 30 s gemessen und mit Normalprobanden verglichen. Auch hier wurden meist topische Anästhetika benutzt, um den Lidschlag zu unterdrücken. Die Mittelwerte der funktionellen Sehschärfe, erhoben mit dem FVA-System, waren zu jedem Zeitpunkt niedriger als bei Kontrollen [25, 33]. Die funktionelle Sehschärfe korrelierte mit dem Oberflächenschaden bei Trockenem Auge [26]. Die Weiterentwicklung, das FVAM-System (Functional Visual Acuity Measurement-System, AS-28, Kowa, Aichi, Japan) wurde im Verlauf in einer Reihe von Studien zur Messung der funktionellen Sehschärfe eingesetzt. Es arbeitet ebenfalls mit Landolt-Optotypen an einem Bildschirm, aber ohne topische Anästhetika bei natürlichem Lidschlag, meist über 60 s $[25,26]$. Lidschläge werden mit diesem System automatisch erfasst [24]. Auch mit diesem System bestätigte sich der Abfall der funktionellen Sehschärfe über Zeit bei Patienten mit Trockenem Auge und die Korrelation zu klinischen Zeichen des Trockenen Auges [26]. Das FVAM-System wurde auch zum Screening für das Trockene Auge bei Menschen mit Computerarbeitsplätzen in der OsakaStudie [28] und der Moriguchi-Studie [34] erfolgreich eingesetzt.

\section{Prüfung der optischen Qualität des visuellen Systems}

\section{Aberrationen höherer Ordnung}

Denoyer et al. führten serielle Messungen der okulären und kornealen Aberrationen höherer Ordnung nach einem Lidschlag für $10 \mathrm{~s}$ durch. Sämtliche Aberrationen höherer Ordnung, v.a. die Aberrationen dritter Ordnung waren signifikant über die 10-s-Periode im Vergleich zu Kontrollprobanden erhöht. Die sog. „modulation transfer function“ (MTF) zeigte eine progrediente Degradation der optischen Qualität, verursacht durch den Kontrastverlust bei intermediären und hohen Ortsfrequenzen bei Patienten mit Trockenem Auge. Die Zunahme an kornealen Aberrationen höherer Ordnung korrelierte mit den subjektiven Symptomen und den objektiven Zeichen des Trockenen Auges im Tränenfilm und an der Augenoberfläche [5]. Eine Zunahme der Aberrationen höherer Ordnung ist somit ein gutes Korrelat für einen Verlust an funktioneller Sehschärfe.

TSAS (Tear Stability Analysis System [Tomey, Nagoya, Japan])

Die Hornhautregularität kann mit einem kornealen Topographiesystem evaluiert werden. Wichtig sind die Parameter SRI, die SAI und die PVA (,potential visual acuity“). SRI ist ein Maß für die lokale Regularität innerhalb der zentralen $4,5 \mathrm{~mm}$ der Hornhaut. Der SRI-Index scheint gut mit der funktionellen Sehschärfe zu korrelieren. Der SAI-Index, ist das Maß für den Unterschied in der Hornhautbrechkraft zwischen 2 Punkten auf dem gleichen Ring 180 Grad voneinander entfernt und somit ein Maß für die Asymmetrie der Hornhaut. Sowohl SRI und SAI sind beim Trockenen Auge erhöht, die potenzielle Sehschärfe ist verringert [35, 36]. Bei kinetischer Hornhauttopographie kann die nichtinvasive Tränenfilmaufreißzeit als Maß für die Tränenstabilität erfasst werden. Das „Tear Stability Analysis System“ (TSAS [Tomey, Nagoya, Japan]) nimmt 10 kontinuierliche HH-Topographiebilder in 10 s auf [37]. Mit dem TSAS-System fanden sich erhöhte SRI- und SAI-Werte bei Patienten mit Trockenem Auge im Zeitverlauf. Die Tränenfilm-Stabilitäts-Indizes TSRI (Tear Stability Regularity Index) und TSAI (Tear Stability Asymmetry Index) waren beide bei Patienten mit Trockenem Auge signifikant erhöht [38]. Patienten mit abnormalen Werten im TSAS-System zeigten ein erhöhtes Risiko für die Entwicklung einer Oberflächenstörung nach LASIK, und ihr Ansprechen auf Therapie war langsamer als bei Patienten mit normaler TSAS-Untersuchung [37].

\section{Optical Quality Analysis System (OQAS)}

Das Optical Quality Analysis System (OQASII, Visiometrics SL, Barcelona, Spanien) wurde in die klinische Praxis eingeführt zur objektiven Messung der optischen Qualität des Auges. Die dynamische Visusqualität wird mit einer Laserdiode von $780 \mathrm{~nm}$ Wellenlänge 
mit einem doppelten Strahlengang („double pass effect“) gemessen als der "objective scatter index (OSI)“. Der OSI ist ein Maß für die Menge an Licht, die bei der Passage durch die brechenden Medien gestreut wird, und somit ein Maß für die optische Qualität des Auges. Der OSI wird nach einem Lidschlag alle $0,5 \mathrm{~s}$ über $20 \mathrm{~s}$ bei geöffnetem Auge ohne Lidschlag oder bei freiem Lidschlag gemessen [39]. Zusätzlich werden die Lidschlagdaten analysiert, und eine sog. „tolerant limitation“ (TL) als Dauer der längsten Interblinkperiode wird errechnet. Der Tränenfilm hat einen großen Einfluss auf die Lichtstreuung durch die Änderung des refraktiven Index zwischen Luft und Tränen. Dementsprechend wurden bei Patienten mit Trockenem Auge signifikant höhere Werte von OSI und OSI/Zeiteinheit gemessen. Die Lidschlagrate war signifikant erhöht und die TL-Dauer signifikant kürzer als bei Kontrollprobanden [39, 40]. Der mittlere OSI korrelierte mit der Schwere des Trockenen Auges [41], der OSI/Zeit mit dem Anfärbegrad der Hornhaut mit Fluoreszein [40].

\section{Einfluss der Therapie auf die funktionelle Sehschärfe}

\section{Tränenersatz}

Eine Reihe von Studien attestiert die positive Wirkung von Tränenersatzmitteln auf die optische Qualität des Auges bei Patienten mit Trockenem Auge. Nach Applikation von Tränenersatz kam es zur signifikanten Verbesserung der Kontrastsensibilität [10, 35, 42] sowie des Ocular Scatter Index (OSI) in der OQAS-Messung [39]. Parameter wie SRI, SAI und mittlerer Astigmatismus reduzierten sich signifikant in der Hornhauttopometrie nach Tränenersatztherapie, und die potenzielle Sehschärfe stieg an [35, 36]. Ein irreguläres topographisches Muster reduzierte sich von $42,2 \%$ der Augen auf $31 \%$ nach der Instillation von Tränenersatz [36]. Die Applikation von Diquafosol führte zur Verbesserung der Tränenfilmstabilität und zum Anstieg des funktionellen Visus gemessen mit dem FVA-System [43]. Aberrationen höherer Ordnung in der Wellenfrontanalyse sinken im Mittel um den Faktor 2,5 nach Tränenersatz [44]. Allerdings können Aberrationen höherer Ordnung (HOA) auch kurzfristig nach Applikation von viskösen Tränenersatzmitteln wie $0,3 \%$ Hyaluronsäure, $3 \%$ Diquafosol oder $2 \%$ Rebamipid ansteigen [45].

\section{Punctum Plugs}

Auch die Insertion von Punctum Plugs kann zu einer Verbesserung der okulären Befunde beim Trockenen Auge führen. Eine Reihe von Arbeiten bestätigt, dass die Therapie mit Punctum Plugs auch die funktionelle Sehschärfe verbessert. Eine signifikante Zunahme der funktionellen Sehschärfe nach Punctum-Plug-Installation wurde v. a. mit den in Japan gebräuchlichen
FVA-Test-Systemen beschrieben [25, 33, 46]. Das Einsetzen von Punctum Plugs war auch mit einer signifikanten Verbesserung der Topographiewerte SRI und SAI assoziiert [38].

\section{Zusammenfassung}

Die funktionelle Sehschärfe ist beim Trockenen Auge reduziert und verbessert sich durch therapeutische Maßnahmen. Mögliche Untersuchungen zur Evaluierung der funktionellen Sehschärfe umfassen die Kontrastsensitivität, die Kontrastsensitivität bei Gegenlicht, die korneale Topometrie im Zeitverlauf, die sequenzielle Wellenfrontanalyse, die Messung der Reduktion der Sehschärfe zwischen 2 Lidschlägen (Interblink Visual Acuity Decay), die Erhebung des funktionellen Visus mit speziellen FVA(,functional visual acuity“)-Testgeräten und die Analyse der Lichtstreuung durch das Optical Quality Analysis System. Derzeit ist die Erhebung der funktionellen Sehschärfe leider noch nicht Teil der Routinediagnostik bei Patienten mit Trockenem Auge.

Funding Open Access funding enabled and organized by Projekt DEAL.

Interessenkonflikt E.M. Messmer gibt an, dass kein Interessenkonflikt besteht.

Open Access Dieser Artikel wird unter der Creative Commons Namensnennung 4.0 International Lizenz veröffentlicht, welche die Nutzung, Vervielfältigung, Bearbeitung, Verbreitung und Wiedergabe in jeglichem Medium und Format erlaubt, sofern Sie den/die ursprünglichen Autor(en) und die Quelle ordnungsgemäß nennen, einen Link zur Creative Commons Lizenz beifügen und angeben, ob Änderungen vorgenommen wurden.

Die in diesem Artikel enthaltenen Bilder und sonstiges Drittmaterial unterliegen ebenfalls der genannten Creative Commons Lizenz, sofern sich aus der Abbildungslegende nichts anderes ergibt. Sofern das betreffende Material nicht unter der genannten Creative Commons Lizenz steht und die betreffende Handlung nicht nach gesetzlichen Vorschriften erlaubt ist, ist für die oben aufgeführten Weiterverwendungen des Materials die Einwilligung des jeweiligen Rechteinhabers einzuholen.

Weitere Details zur Lizenz entnehmen Sie bitte der Lizenzinformation auf http://creativecommons.org/licenses/by/4. $0 /$ deed.de.

\section{Literatur}

1. No authors listed. The definition and classification of dry eye disease: report of the definition and classification subcommittee of the international dry eye workshop (2007). OculSurf. 2007;5:75-92.

2. CraigJP,NicholsKK,AkpekEK, etal.TFOSDEWSIIdefinition and classification report. Ocul Surf. 2017;15:276-83.

3. Goto E, Yagi Y, Matsumoto Y, Tsubota K. Impaired functional visual acuity of dry eye patients. Am J Ophthalmol. 2002;133:181-6. 
4. Koh S. Irregular astigmatism and higher-order aberrations in eyes with dry eye disease. Invest Ophthalmol Vis Sci. 2018;59:DES36-DES40.

5. Denoyer A, Rabut G, Baudouin C. Tear film aberration dynamics and vision-related quality of life in patients with dry eye disease. Ophthalmology. 2012;119:1811-8.

6. Montés-Micó R, Cáliz A, Alió JL. Wavefront analysis of higher order aberrations in dry eye patients. J Refract Surg. 2004;20:243-7.

7. KohS. Mechanisms of visual disturbancein dry eye. Cornea. 2016;35(1):S83-S8.

8. Shimazaki-DenS, Dogru M, Higa K, ShimazakiJ.Symptoms, visual function, and mucin expression of eyes with tear film instability. Cornea. 2013;32:1211-8.

9. Ridder WH 3rd, Tomlinson A, Huang JF, Li J. Impaired visual performance in patients with dry eye. Ocul Surf. 2011;9:42-55.

10. Rolando M, Iester M, Macrí A, Calabria G. Low spatialcontrast sensitivity in dry eyes. Cornea. 1998;17:376-9.

11. Benítez-Del-Castillo J, Labetoulle M, Baudouin C, et al. Visual acuity and quality of life in dry eye disease: proceedings of the OCEAN group meeting. Ocul Surf. 2017;15:169-78.

12. Barber L, Khodai O, Croley T, et al. Dry eye symptoms and impact on vision-related function across international task force guidelines severity levels in the United States. BMC Ophthalmol.2018;18:260.

13. Walker PM, Lane KJ, Ousler GW 3rd, Abelson MB. Diurnal variation of visual function and the signs and symptoms of dry eye. Cornea. 2010;29:607-12.

14. Ousler GW 3rd, Abelson MB, Johnston PR, Rodriguez J, Lane K, Smith LM. Blink patterns and lid-contact times in dryeye and normal subjects. Clin Ophthalmol. 2014;8:869-74.

15. Doga AV, Maychuk NV, Mushkova IA, Shamsetdinova LT. Causes, prevention and correction of refractive errors after phacoemulsification with intraocular lens implantation. Vestn Oftalmol.2019;135:83-90.

16. Epitropoulos AT, Matossian C, Berdy GJ, Malhotra RP, Potvin R. Effect of tear osmolarity on repeatability of keratometry for cataract surgery planning. J Cataract Refract Surg. 2015;41:1672-7.

17. Starr CE, Gupta PK, Farid M, et al. An algorithm for the preoperative diagnosis and treatment of ocular surface disorders. JCataract RefractSurg. 2019;45:669-84.

18. Szakáts I, Sebestyén M, TóthÉ, PureblG. Dry eye symptoms, patient-reported visual functioning, and health anxiety influencing patient satisfaction after cataract surgery. Curr Eye Res. 2017;42:832-6.

19. Karakus S, Mathews PM, Agrawal D, Henrich C, Ramulu PY, Akpek EK. Impact of dry eye on prolonged reading. Optom Vis Sci. 2018;95:1105-13.

20. Ridder WH 3rd, Zhang Y, Huang JF. Evaluation of reading speed and contrast sensitivity in dry eye disease. Optom Vis Sci. 2013;90:37-44.

21. Karakus S, Agrawal D, Hindman HB, Henrich C, Ramulu PY, Akpek EK. Effects of prolonged reading on dry eye. Ophthalmology. 2018;125:1500-5.

22. Mathews PM, Ramulu PY, Swenor BS, Utine CA, Rubin GS, Akpek EK. Functional impairment of reading in patients with dry eye. Br JOphthalmol. 2017;101:481-6.

23. Deschamps N, Ricaud X, Rabut G, Labbé A, Baudouin C, Denoyer A. The impact of dry eye disease on visual performance while driving. Am J Ophthalmol. 2013;156:184-189.e3.

24. Kaido M. Functional visual acuity. Invest Ophthalmol Vis Sci. 2018;59:DES29-DES35.
25. Kaido M, Dogru M, Ishida R, Tsubota K. Concept of functional visual acuity and its applications. Cornea. 2007;26:S29-35.

26. Kaido M, Ishida R, Dogru M, Tsubota K. The relation of functional visual acuity measurement methodology to tear functions and ocular surface status. Jpn J Ophthalmol. 2011;55:451-9.

27. Kaido M, Matsumoto Y, Shigeno Y, Ishida R, Dogru M, Tsubota K. Corneal fluorescein staining correlates with visual function in dry eye patients. Invest Ophthalmol Vis Sci. 2011:52:9516-22.

28. Kaido M, Uchino M, Yokoi N, et al. Dry-eye screening by using a functional visual acuity measurement system: the Osaka study. Invest Ophthalmol Vis Sci. 2014;55:3275-81.

29. McAlinden C, Pesudovs K, Moore JE. The development of an instrument to measure quality of vision: the quality of vision (QoV) questionnaire. Invest Ophthalmol Vis Sci. 2010;51:5537-45.

30. Szczotka-Flynn LB, Maguire MG, Ying GS, et al. Impact of dry eye on visual acuity and contrast sensitivity: dry eye assessment and management study. Optom Vis Sci. 2019;96:387-96.

31. Puell MC, Benítez-del-Castillo JM, Martínez-de-la-Casa J, etal. Contrast sensitivity and disability glare in patients with dry eye. Acta OphthalmolScand. 2006;84:527-31.

32. Torkildsen G. The effects of lubricant eye drops on visual function as measured by the inter-blink interval visual acuity decay test. Clin Ophthalmol. 2009;3:501-6.

33. Ishida R, Kojima T, Dogru M, et al. The application of a new continuous functional visual acuity measurement system in dry eye syndromes. Am J Ophthalmol. 2005;139:253-8.

34. Kaido M, Kawashima M, Yokoi N, et al. Advanced dry eye screening for visual display terminal workers using functionalvisualacuitymeasurement: the Moriguchistudy. BrJOphthalmol.2015;99:1488-92.

35. Huang FC, Tseng SH, Shih MH, Chen FK. Effect of artificial tears on corneal surface regularity, contrast sensitivity, and glare disability in dry eyes. Ophthalmology. 2002;109:1934-40.

36. Liu Z, Pflugfelder SC. Corneal surface regularity and the effect of artificial tears in aqueous tear deficiency. Ophthalmology. 1999;106:939-43.

37. Goto T, Zheng X, Okamoto S, Ohashi Y. Tear film stability analysis system: introducing a new application for videokeratography. Cornea. 2004;23:S65-70.

38. Kojima T, Ishida R, Dogru M, et al. A new noninvasive tear stability analysis system for the assessment of dry eyes. Invest Ophthalmol Vis Sci. 2004;45:1369-74.

39. Diaz-Valle D, Arriola-Villalobos P, García-Vidal SE, et al. Effect of lubricating eyedrops on ocular light scattering as a measure of vision quality in patients with dry eye. JCataract Refract Surg. 2012;38:1192-7.

40. Tan CH, Labbé A, Liang Q, et al. Dynamic change of optical quality in patients with dry eye disease. Invest Ophthalmol Vis Sci. 2015;56:2848-54.

41. Habay T, Majzoub S, Perrault O, Rousseau C, Pisella PJ. Objective assessment of the functional impact of dry eye severityon the quality of vision bydouble-pass aberrometry. JFr Ophtalmol. 2014;37:188-94.

42. Ridder WH 3rd, Tomlinson A, Paugh J. Effect of artificial tears on visual performance in subjects with dry eye. Optom Vis Sci. 2005;82:835-42.

43. Kaido M, Uchino M, KojimaT, Dogru M, Tsubota K. Effects of diquafosol tetrasodium administration on visual function in short break-up time dry eye. J Ocul Pharmacol Ther. 2013;29:595-603. 
44. Montés-Micó R, Cerviño A, Ferrer-Blasco T, GarcíaLázaro S, Ortí-Navarro S. Optical quality after instillation of eyedrops in dry-eye syndrome. J Cataract Refract Surg. 2010;36:935-40.

45. Koh S, Maeda N, Ikeda C, et al. Effect of instillation of eyedrops for dry eye on optical quality. Invest Ophthalmol Vis Sci.2013;54:4927-33.
46. Goto E, Yagi Y, Kaido M, Matsumoto Y, Konomi K, Tsubota K. Improved functional visual acuity after punctal occlusion in dry eye patients. Am J Ophthalmol.2003;135:704-5.

Hinweis des Verlags Der Verlag bleibt in Hinblick auf geografische Zuordnungen und Gebietsbezeichnungen in veröffentlichten Karten und Institutsadressen neutral. 